\section{Intersections}

Canadian Journal of Music

Revue canadienne de musique
Intersections CANADIAN JOURAL OF MUSIC
REVUE CANADIENEE DE MUSIOUH

\title{
Le geste sonore comme objet d'analyse pour observer les pratiques musicales distinctives d'improvisateurs jazz
}

\section{Martin Desjardins}

Volume 37, numéro 2, 2017

URI : https://id.erudit.org/iderudit/1066617ar

DOI : https://doi.org/10.7202/1066617ar

Aller au sommaire du numéro

\section{Éditeur(s)}

Canadian University Music Society / Société de musique des universités canadiennes

\section{ISSN}

1911-0146 (imprimé)

1918-512X (numérique)

Découvrir la revue

Citer cet article

Desjardins, M. (2017). Le geste sonore comme objet d'analyse pour observer les pratiques musicales distinctives d'improvisateurs jazz. Intersections, 37(2), 43-58. https://doi.org/10.7202/1066617ar

\section{Résumé de l'article}

Outre le choix des notes, qui est prescrit par notre tradition commune, qu'est-ce qui distingue une ou un improvisateur jazz d'une ou un autre ? Qu'est-ce qui constitue leur "son ", leur " signature " ? Les méthodes d'analyses plus traditionnelles n'étant pas suffisantes pour circonscrire l'entièreté de l'expression contenue dans l'improvisation jazz, nous devons nous tourner vers l'étude d'enregistrements de solos improvisés afin d'examiner l'aspect « performanciel » de la musique, c'est-à-dire les éléments liés à la production du son par l'instrumentiste. Cependant, l'oreille pouvant omettre certains éléments au profit d'autres, nous avons adapté un modèle d'analyse par spectrogramme utilisé pour l'analyse du chant en musique populaire. En combinant le visuel à l'auditif, ce modèle permet d'effectuer un inventaire plus exhaustif des " gestes sonores " contenus dans les improvisations, c'est-à-dire toutes manifestations sonores produites par des gestes instrumentaux effectués au sein d'un processus musical. À travers un projet de recherche-création, une méthode autoethnographique a permis de distinguer des gestes sonores dans mes propres improvisations et ainsi de les comparer avec celles du saxophoniste de réputation internationale Chris Potter. Les analyses distinguent cinq gestes sonores, subtils mais distinctifs, observés à partir des micro-variations de hauteurs lors de l'attaque ou le déclin de certaines notes, de l'utilisation particulière de sons multiphoniques, du vibrato, des jeux de dynamiques et de bruits de clés.
Copyright @ Canadian University Music Society / Société de musique des universités canadiennes, 2019
Ce document est protégé par la loi sur le droit d'auteur. L’utilisation des services d'Érudit (y compris la reproduction) est assujettie à sa politique d'utilisation que vous pouvez consulter en ligne.

https://apropos.erudit.org/fr/usagers/politique-dutilisation/ 


\title{
LE GESTE SONORE COMME OBJET D'ANALYSE POUR OBSERVER LES PRATIQUES MUSICALES DISTINCTIVES D'IMPROVISATEURS JAZZ ${ }^{1}$
}

\author{
Martin Desjardins
}

Dans l'improvisation jazz, le corps et ses manifestations font partie intégrante de la pratique (Béthune 1991; Mouëllic 2000; Cotro 2014). L'expression du musicien ne se situe pas que dans les structures formelles de la musique, qu'elle soit écrite, paraphrasée ou improvisée, mais aussi, peut-être même surtout, dans les manifestations sonores du corps musiquant (Cotro 2014, 66). Il est vrai que toute forme de production sonore instrumentiste possède des caractéristiques uniques, propres à la musicienne ou au musicien en prestation. En effet, pour une partition donnée, la prestation d'une personne sera discernable de celle d'une autre et ce, même si un respect tatillon du texte musical est appliqué. Ainsi, les traits constitutifs d'une création sont ceux de la partition tandis que les traits contingents sont propres à une prestation donnée et aux caractéristiques personnelles de l'instrumentiste (timbre et effets de timbre, sons, bruits, etc.). On parle alors de deux régimes d'immanence de la création musicale: l'allographie pour les éléments que l'on peut écrire, la création dans sa forme idéale, et l'autographie pour la réalisation actuelle, sonore de cette forme idéale (Genette 1994 cité par Lacasse 2006, 68-71).

Cependant, en improvisation, cette forme idéale ne peut être préalablement écrite, confondant ainsi les éléments allographiques et autographiques. En effet, l'improvisation est une création extemporanée, c'est-à-dire qu'elle est par essence constituée d'éléments non préalablement écrits. Si elle possède systématiquement un "point de départ» (Ford 1995, 83; Nettl 1998, 12-19; 2003, 406; Nettl et al., s.d., section 1.3), aussi appelé «modèle» (Lortat-Jacob 1987, 54-57; Siron 2012,79$)^{2}$, qui établit les règles qui encadrent l'improvisation, cette forme idéale ne génère en soi aucun résultat analysable et ne constitue que le seul repère identifiable d'une pensée de départ. Au contraire, issue de l'actualisation de la pensée par le geste, l’acte de «musiquer» (Béthune 2009) transforme

1 Cet article a reçu le prix de la Fondation SOCAN / MUSCAN pour la recherche sur la musique canadienne. Il est également disponible sur le site web de MusCan: http://muscan.org/fr/

2 Les «règles par la négative: pas de tempo, pas de tonalité, pas de hiérarchie, pas de beau son, etc.» (Siron 2007, 700) du free jazz sont de règles en soi. De même que de déclarer l'absence de règle pour l'improvisation libre. De plus, pour cette forme d'improvisation, chaque instrumentiste amène son propre bagage, constituant un point de départ par défaut. 
la pensée de départ et génère un résultat différent de cette idée. Ainsi, s'il pouvait être avancé que la forme idéale de l'improvisation est cette idée de départ, le corps de l'improvisateur s'impose dans le processus d'actualisation par le geste, qui peut être inconscient, voire accidentel, mais non moins significatif pour l'improvisateur ou l'auditeur. Donc, l'esprit et le corps sont solidaires dans cette forme d'expression musicale et la forme idéale d'une improvisation est inextricablement enchevêtrée avec la performance.

À cause de cette solidarité du corps et de l'idée, l'improvisation est un état particulier de la création musicale. De cette façon, les éléments constitutifs et contingents se retrouvent à être intimement liés, au point d'être indissociable les uns des autres:

[1]'improvisation n'est [...] pas un état plus pur des arts de performance, mais au contraire un état plus complexe, où se mêlent, de façon souvent inextricable en pratique, deux œuvres théoriquement distinctes: un texte (poétique, musical ou autres) susceptible de notation après coup et de multiplication indéfinie, qui en constitue le versant allographique, ou idéal, et une action physique (autographique) dont les caractéristiques matérielles ne peuvent être intégralement notées, mais peuvent en revanche être imitées, voire contrefaites. (Genette 1994 cité par Lacasse 2006, 71)

Autrement dit, la «forme idéale» d'une improvisation ne sera jamais quelque chose que l'on pourra examiner via une représentation, ni a priori (ce qui reviendrait à tenter de lire dans les pensées), ni a posteriori. Au mieux, peut-on après-coup consulter un enregistrement - qui est une représentation de l'improvisation et non l'improvisation elle-même - noter avec une précision relative les éléments dits formels que sont la hauteur, le rythme, l'harmonie, etc., et tenter de reproduire «à l'oreille» le résultat sonore. Ainsi, dans une improvisation, les deux régimes d'immanence de la création musicale se doivent d'être observés ensemble, puisqu'ils sont exprimés en même temps et que le sens qui en émane ne peut être constaté qu'à partir d'une analyse globale des caractéristiques sonores de la performance.

À la suite de Desroches (2008), nous adoptons une définition de performance proche du concept de pragmatique élaboré par les linguistes qui se décrit comme: «tout ce qui intervient dans l'interprétation d'un énoncé, mais qui n'est traité directement ni par la syntaxe, ni par la sémantique» (Monod-Becquelin et Vapnarsky 2001, 161 cité par Desroches 2008, 103-104). De cette façon, dans la communication verbale, performance désigne d'une part les gestes faisant partie intégrante de la communication: « [g]esture produced in relation to speech are an integral component of the communicative act of the speaker [...] they must be seen as part of the speaker final product» (Kendon 2004, 359). De cette façon, le geste est porteur de sens et est co-expressif avec la parole (McNeill 2008, 22). D'autre part, ce concept de performance comprend le paralangage, c'est-à-dire la façon dont cette parole est exprimée par l'entremise d'effets sonores qui apparaissent en même temps que les mots et qui participent aussi à l'acte de communication. La conjonction des mots, des gestes visibles et des 
effets sonores constitue la «triple structure de base de la communication faceà-face: langage-paralangage-cinétique» (Poyatos 1993, chap. 3$)^{3}$.

L'improvisation musicale partage avec la communication face-à-face entre deux ou plusieurs personnes le caractère extemporané des interactions directes, un «point de départ» où l'on retrouve, dans le cas des improvisations idiomatiques, une syntaxe musicale, ainsi qu'un ensemble de gestes visibles et d'effets sonores qui participent à la communication. Autrement dit, on peut identifier la même «triple structure de base» en communication orale qu'en improvisation musicale. Cependant, dans le musical, le concept de performance tient lieu de paralangage et justifie l'utilisation de l'adjectif "performanciel» pour désigner: «tout aspect de la musique vocale ou instrumentale relevant de la production de sons par un musicien» (Lacasse 2006 cité par Desroches 2008, 105). L'étude des paramètres performanciels met non seulement en évidence les aspects sonores de la production du musicien, mais il témoigne également de façons de faire qui participent significativement à la stylistique d'une pratique musicale. Par exemple, un tambourineur martiniquais de la commune de Sainte-Marie ne jugera pas une performance par sa réussite à reproduire un rythme abstrait, mais sur les couleurs sonores produites par un coup de poignet adéquat à l'exécution de ce rythme (Desroches 2014, 291). Par extension, l'étude de la performance permet d'identifier la «signature singulière» (Desroches 2008) d'un musicien, c'est-à-dire les manières particulières dont il joue les notes, chante ou frappe la peau d'un tambour, les caractéristiques sonores qui le distinguent de tous les autres instrumentistes. Ainsi, l'étude des paramètres performanciels permettrait de discerner les traits caractéristiques d'un improvisateur en analysant les éléments de performance contenus dans ses improvisations.

L'analyse musicale ne pouvant se faire seulement à partir des paramètres compositionnels, elle doit également tenir compte des paramètres phonographiques et des paramètres performanciels (Lacasse 2006). Comme l'improvisation est spontanée, la création apparaît sur le moment et laisse entendre en même temps les éléments formels et performanciels. De cette façon, les actions réalisées par l'improvisateur sont indissociables des éléments conceptuels qu'il laisse entendre. Ainsi pour mieux comprendre la prestation du musicien jazz, l'analyste doit absolument tenir compte des paramètres performanciels, c'està-dire observer «[le] geste du musicien en plein acte de création» (Stévance 2011, 53).

Le geste se définit comme un «mouvement limité ou ébauche de mouvement du corps, de la tête, des membres, des mains, ayant une signification symbolique (par exemple: le clignement d'un œil, un 'au-revoir') ${ }^{4}$ : «Bien que le geste soit aussi visible que le mouvement ou l'action, il a la particularité d'être expressif, tout comme le langage duquel il se différencie fondamentalement» (Héroux et Fortier 2014, 22). À cet égard, le mouvement représente

3 Notre traduction de «The Basic Triple Structure of Communication in Face-To-Face interaction: Language-Paralanguage-Kinetics».

4 Geste. 1973. Dans Le grand dictionnaire terminologique de l'Office québécois de la langue française. Repéré à http://www.granddictionnaire.com/ficheOqlf.aspx?Id_Fiche=17588618 
un changement qui peut être objectivement mesuré dans la position du corps, d'une partie de celui-ci, ou même d'un objet (Dahl et al. 2010), tandis que l'action se définit comme un agrégat cohérent de mouvements ou de gestes toujours réalisés de manière intentionnelle et dirigés vers un objectif (Jensenius et al. 2010). Donc, le geste, ici, se distingue du mouvement et de l'action par sa signification symbolique et son caractère expressif.

Le geste communique du sens, tout comme les mots, mais n'est pas dépendant de ceux-ci. Parce qu'il exprime à la fois des caractéristiques physiques et expressives, c'est à partir des gestes, plutôt que par l'étude du mouvement ou de l'action, que l'on peut observer à la fois les caractéristiques constitutives et contingentes d'une performance musicale. En effet, la notion de geste amalgame le mouvement, défini comme le déplacement d'un objet dans l'espace, et le sens, c'est-à-dire l'activation mentale d'une expérience (Hatten 2003 dans Jensenius et al. 2010, 18). Donc, sous cette définition, la notion de geste surpasse la division entre corps et esprit, entre l'idée et l'actualisation de cette idée. C'est pourquoi le geste musical semble l'objet idéal à l'analyse de la performance.

Pourtant, cette distinction du corps et de l'esprit semble refaire surface dans la façon dont la musicologie aborde les gestes musicaux. En effet, le geste sonore aurait plusieurs niveaux, de purement fonctionnel à purement symbolique qui se déclinent en gestes « effectifs» c'est-à-dire strictement relatifs à la production du son, en gestes «accompagnateurs» c'est-à-dire qui accompagnent les gestes effectifs, ainsi que les gestes « figuratifs » c'est-à-dire qui peuvent communiquer une image mentale strictement par le son, sans être visibles (Delalande 1988). De plus, dans ce paradigme, les gestes accompagnateurs «supportent» les gestes effectifs lorsqu'ils sont audibles et l'«accompagnent» lorsqu'ils sont nonaudibles (Héroux et Fortier 2014). Bien que déclinés sous différentes terminologies, les gestes musicaux étudiés tombent sous l'une ou l'autre de ces fonctions. Ainsi, les gestes relatifs à la production du son sont dits instrumental (Cadoz et Wanderley 2000) ou sound producing (Jensenius et al. 2010; Godøy 2011), les gestes qui supportent la production du son sont dits non-obvious (Wanderley 1999), ancillary (Jensenius et al. 2010; Wanderley et Depalle 2004) ou soundtracing (Godøy 2011), les gestes qui accompagnent la prestation, sans mener au sonore sont dits sound accompanying (Jensenius et al. 2010) ou theatrical (Godøy 2011), et les gestes pour la communication sont appelés communicative (Jensenius et al. 2010) ou semiotic gestures (Cadoz et Wanderley 200o). Malgré leur finesse, ces observations entretiennent les distinctions cartésiennes en établissant que certains gestes expriment seulement une idée, d'autres la forme et enfin que les autres «accompagnent» ou «supportent» la production d'un son, hiérarchisant ainsi les différents types de gestes musicaux. Pourtant, c'est ici une question de perception issue de notre culture musicale: «[a]ccompaniment can only occur within a musical structure consisting of separate strands exhibiting different degrees of perceived importance» (Tagg 2000, 17) $)^{5}$.

Ces distinctions semblent inévitables tant que l'on en reste à départager le musical du sonore. De cette façon, le problème serait ontologique et résulterait

5 Les italiques sont de moi. 
de notre conception même de ce qu'est le musical et de ce qui en est exclu. Cependant, si l'on inclut dans le musical tout son, c'est-à-dire les potentialités du timbre, du bruit et de l'espace comme éléments musicaux à part entière (Solomos 2013), il n'est plus possible d'examiner le geste musical de la même façon, du moins lorsque celui-ci est audible. En effet, si on ne s'en tient qu'au sonore, les gestes musicaux audibles apparaissent plutôt comme des agrégats coarticulés dont les fonctions sont à la fois formelles et expressives. Ainsi, pourrait-on observer des «objets sonores coarticulés» (Godøy 2011), définis comme des unités issues de la convergence du son et du mouvement, des agrégats perçus comme un tout.

Malgré qu'ils soient perçus comme un tout, ces «objets sonores coarticulés» n'incluent pas tous les aspects du sonore. En effet, il semble, selon cette définition, que l'on devrait exclure le "smearing» (que l'on peut traduire par barbouillage ou salissure), c'est-à-dire des éléments sonores qui, comme leur nom l'indique, semblent indésirables (Godøy 2011). De cette façon, on retombe encore une fois dans la distinction entre le musical et le sonore, établissant une séparation qui ne peut qu'être que catégorique entre un esprit qui cherche à exprimer une idée "propre» et un corps qui la «salie».

Ainsi, la performance en improvisation jazz instrumentale s'observe à partir des gestes musicaux audibles produits par l'instrumentiste, puisque ceuxci présentent à la fois les traits constitutifs et contingents de la création. Ces gestes relèvent des paramètres performanciels contenus dans une prestation et leur examen rigoureux permettrait peut-être de distinguer la stylistique particulière, les traits distinctifs d'instrumentistes improvisateurs. Cependant, cet examen nécessite de tenir compte de tout le sonore contenu dans ces gestes, mais le paradigme musicologique plus traditionnel où l'on doit distinguer le sonore du musical conditionne encore aujourd'hui bien des recherches sur le sujet. De cette façon, nous nous demanderons en quoi l'examen des paramètres performanciels permet la distinction de pratiques musicales chez des improvisateurs jazz.

Cet article a comme objectifs l'identification des gestes musicaux qui incluent tout le domaine du sonore, ainsi que l'observation des aspects de la performance qui distingue la stylistique de différents improvisateurs.

Dans une perspective qui inclut le sonore en tant qu'élément musical à part entière, le geste musical réfère à la performance, c'est-à-dire aux processus somatiques effectués au sein d'un processus musical (Middleton 1993). De cette façon, le geste musical couvre non seulement les mouvements sonores nettement audibles, mais aussi des microvariations de l'intonation, les articulations et les ajustements timbrales. De plus, le geste musical se voit ici libéré des notions d'intention, de direction syntaxique et de sa fonction symbolique, mais non de sa fonction expressive. Conséquemment, c'est un geste qui renvoie directement à l'expression de l'instrumentiste tout en laissant de côté les préoccupations formelles. De plus, ces manifestations sonores du corps et du processus de production du son par celui-ci permettraient de distinguer un musicien d'un autre: 
L'observation attentive, microscopique, des mouvements associés à la réalisation de la musique, particulièrement la mobilité de ceux impliqués dans la création de la pulsion vitale, l'attention portée à la façon de phraser de chaque participant, le relevé des "gestes sonores» caractéristiques du soliste, en bref, l'emploi de l'approche processuelle, je l'espère, mènera finalement à des résultats plus satisfaisants, intellectuellement et émotionnellement (Keil 1966 cité par Cugny 2009, 344)

Ainsi, l'étude du processus de création sonore par l'observation des gestes, même les plus infimes, qui constituent la performance pourrait nous permettre de distinguer un improvisateur d'un autre. Afin d'éviter toute confusion et comme l'expression "geste musical» renvoie à l'ambiguïté même de ce qui constitue le musical par rapport au sonore, nous reprendrons l'expression de Keil et utiliserons à partir d'ici «geste sonore» pour désigner toutes manifestations sonores produites par des gestes instrumentistes effectués au sein d'un processus musical.

Pour savoir s'il est possible de distinguer un improvisateur jazz grâce aux gestes sonores, nous devrons premièrement les identifier dans les improvisations de deux instrumentistes et deuxièmement, observer si ces gestes montrent des différences qui permettent de distinguer ces deux instrumentistes.

Comme il est impossible d'atteindre nos objectifs par l'analyse d'improvisations en temps réel, ce sont les enregistrements d'improvisations qui peuvent nous fournir les informations nécessaires à nos observations. Bien que l'enregistrement ne soit qu'une représentation de la performance, non un état de celle-ci (Genette 1994), cette représentation peut, avec le matériel adéquat, être une représentation suffisamment fidèle pour l'analyse du jazz (Cugny 2004, 2009; Cottrell 2010). Afin de bien pouvoir identifier les gestes sonores, l'utilisation d'une représentation visuelle en plus de la représentation sonore semble appropriée. Ainsi, nous empruntons et adaptons la méthode d'analyse à partir de spectrogrammes pour la voix en musique populaire (Lacasse 2010). En effet, cet outil permet de visualiser les différents effets, sons, micro-intonations, bref, la façon dont sont chantées les notes, en plus d'illustrer les différents bruits, souffles et variations de timbre. De cette façon, chaque effet a sa représentation spectrographique propre, son «dessin » particulier, qui permet de l'identifier et éventuellement mieux comprendre sa fonction expressive.

Cette technique peut aisément être transposée pour les instruments à vent, vu la proximité entre les manières de produire l'expression. En effet, l'expression vocale et la prestation musicale possèdent plusieurs similarités, particulièrement en ce qui concerne la communication d'émotions (Juslin et al. 2003). Conséquemment, l'expression produite par le lien geste-instrument peut s'étudier de la même façon que le lien geste-voix, notamment grâce à des représentations auditives (l'enregistrement) et visuelles (le spectrogramme).

Tel que vu plus haut, le jazz a composé sa matière sonore et visuelle à partir du corps des instrumentistes, un corps qui s'exprime à partir de gestes visibles et audibles. De ce point de vue, c'est un style musical où on devrait retrouver un grand nombre de gestes sonores. C'est pourquoi il semble approprié pour l'analyse de l'expression chez les instrumentistes. 
À cet égard, les saxophonistes ténors ont exploré de manière étendue, par leur pratique, les textures et les timbres propres à leur instrument:

Dans la musique de jazz, par exemple, il suffit des premières notes d'un saxophone ténor [ ... ] pour savoir de qui il s'agit: Coleman Hawkins, Lester Young, Sonny Rollins ou John Coltrane. Produire des sons révèle un style propre à chaque musicien à utiliser, à bouger et à faire vibrer son propre corps. Il ne s'agit pas de jouer de l'instrument, mais de faire résonner, par le biais de cet instrument, son propre corps (Sparti 2009, 5)

Étant moi-même saxophoniste jazz professionnel depuis plus de 20 ans (selon les définitions du Conseil des arts du Canada et du Conseil des arts et des lettres du Québec), ma propre pratique est une partie du terrain d'investigation. Cette démarche de recherche-création s'inscrit dans la définition proposée par Stévance et Lacasse:

Research-creation is understood as an approach applied to an individual or multiple-agent project combining research methods and creative practices within a dynamic frame of causal interaction (that is, each having a direct influence on the other), and leading to both scholarly and artifactual productions (be they artistic or otherwise) (Stévance et Lacasse 2017, 123)

Ainsi, pour collecter les données issues de ma pratique, l'approche autoethnographique est appropriée. La méthode autoethnographique au sein d'un projet de recherche-création permet une perspective praticienne de première main qui s'ajoute aux observations plus directes. Cependant, pour être valable, cette méthode doit transcender l'autobiographie afin de connecter le personnel et le culturel (Chang 2008). C’est pourquoi les résultats doivent être validés à partir d'autres observations que ceux obtenus par l'examen de ma propre pratique.

Ainsi, en plus de l'analyse de mes propres improvisations, l'examen de créations d'un autre saxophoniste a été effectué. Les données sont recueillies à partir de quatre improvisations: mes deux improvisations ont été faites à partir des schémas harmoniques de deux de mes compositions intitulées Pour FB et Le printemps à l'automne, tandis que les analyses comparatives sont tirées d'improvisations faites à partir de compositions intitulées Act III, scene I et What's New du saxophoniste Chris Potter.

Par l'analyse de ces improvisations, il a été possible de tirer cinq exemples de gestes sonores distinctifs. Un premier geste identifié se produit lors de l'attaque et se reconnait sur le spectrogramme par sa forme de crochet visible au début d'une note. Au son, on entend une note attaquée à une hauteur qui redescend rapidement, puis qui remonte à l'intonation initiale ou plus haut. Si ces attaques sont semblables chez les deux instrumentistes, on repère parfois chez Potter des microvariations de hauteurs qui apparaissent comme des «dents-de-scie» se superposant à la forme habituelle de cette attaque. Cela indique la présence d'un microvibrato coarticulé avec les plus grandes variations de hauteurs qui, à cause de sa rapidité, a un impact sur le timbre plutôt que d'être perçu comme un vibrato. De plus, Potter utilise le même effet à d'autres 


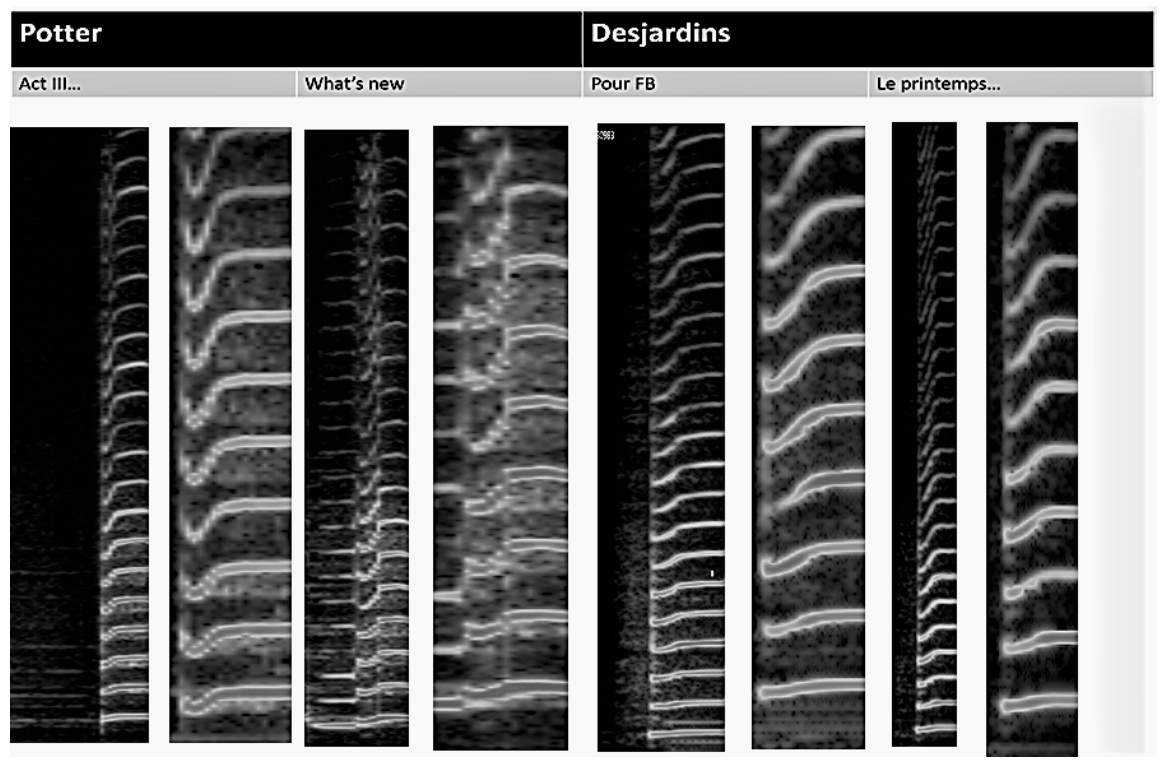

Figure 1. Spectrogrammes illustrant les différences dans l'attaque de certaines notes entre Potter et Desjardins.

moments (par exemple sur des «tombées», c'est-à-dire une note dont l'intonation descend rapidement et de façon évidente) alors que ce geste sonore n'est jamais présent dans mes propres improvisations.

Un autre effet commun retrouvé dans toutes les improvisations est la note multiphonique. Un son multiphonique est caractérisé par la production de plusieurs notes en même temps sur un instrument normalement monophonique. Le phénomène se reconnait à l'oreille par un son qui s'apparente à un cri rauque et aigu, tandis qu'il s'affiche sur le spectrogramme par la présence $\mathrm{d}^{\prime}$ harmoniques plus pâles entre les harmoniques de la note principale ${ }^{6}$. Encore une fois, Potter se distingue en ajoutant souvent un vibrato à la fin de la note alors que dans mes improvisations, la note multiphonique ne subit pas d'autres variations.

Parmi les improvisations observées, le vibrato est un élément distinctif. Perceptible à l'oreille comme une oscillation répétée de la hauteur, il apparait sur le spectrogramme par des ondulations, régulières ou non, des harmoniques. Nous pouvons remarquer que, chez Potter, un vibrato rapide et suffisamment prononcé apparait quasi systématiquement sur de longues séquences de notes jouées et donne à ses deux improvisations une texture distinctive par rapport aux miennes. Étant donné la forte utilisation de cet effet, les improvisations de Potter ont une atmosphère générale plus "chantante» alors que mes improvisations seront plus «lisses», car les notes «vibrées» le sont beaucoup plus

6 En effet, malgré qu'il soit en deux dimensions, le spectrogramme représente trois paramètres: le temps en abscisse, les fréquences hertziennes en ordonnées, ainsi que les couleurs pour l'intensité en décibels FS. 


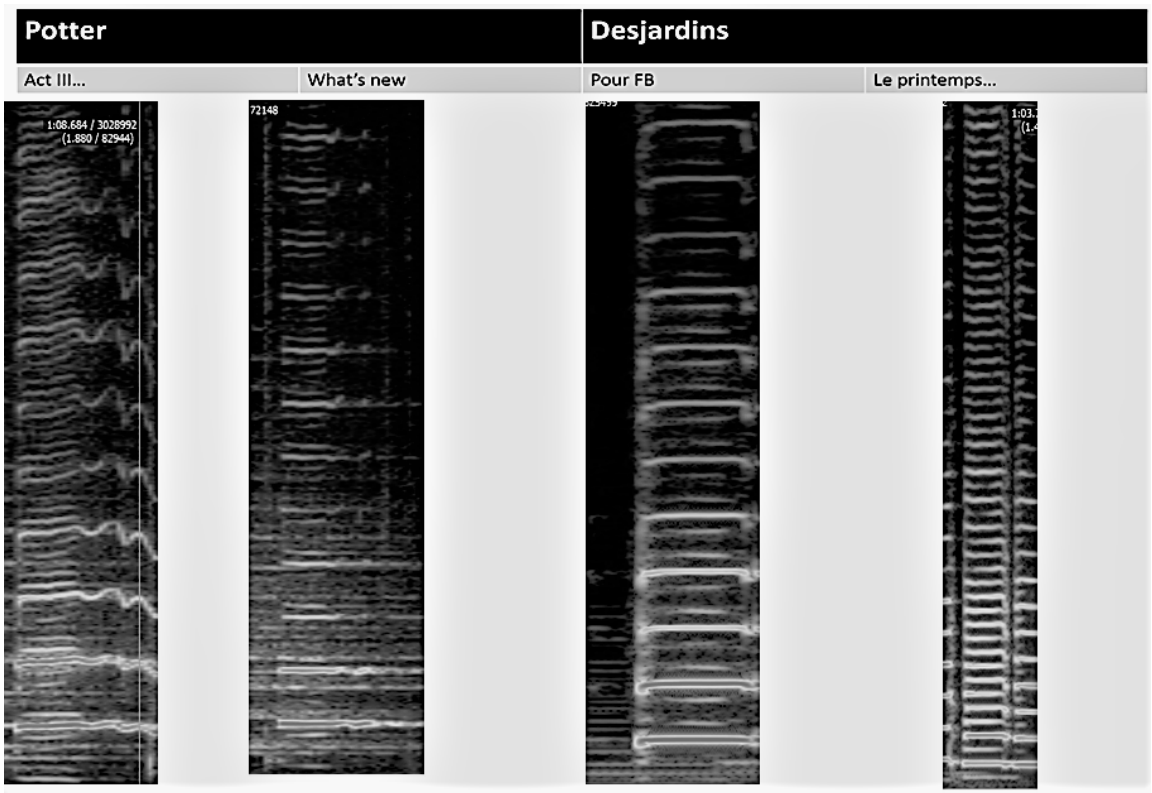

Figure 2. Spectrogrammes illustrant les différences dans l'utilisation de multiphoniques entre Potter et Desjardins.

légèrement et de façon non systématique. Bien sûr, nous pouvons observer des vibratos prononcés et rapides dans mes improvisations, mais la haute récurrence avec laquelle elle apparait chez Potter et la rareté avec laquelle cela se produit chez moi est ce qui nous distingue.

Une autre distinction se fait entendre dans le jeu des nuances. En effet, les deux improvisations de Potter laissent entendre des séquences où des notes au volume doux sont alternées avec d'autres jouées au volume moyen ou fort. Cet effet de contraste donne à ces séquences, ainsi qu'à l'improvisation en général, un caractère dynamique que l'on ne retrouve pas dans mes improvisations qui, à cause de nuances moins prononcées, possèdent un caractère plus constant et linéaire. Sur le spectrogramme, les notes de niveaux moyen et fort présentent des harmoniques bien visibles au-delà de fréquences suraiguës, tandis que les notes plus douces laissent voir une intensité moindre, donc beaucoup moins d'harmoniques aiguës visibles.

Inclure tout le spectre du sonore dans les analyses implique de tenir compte non seulement des sons périodiques, mais aussi les sons non périodiques. Un de ceux-là se retrouve forcément dans les enregistrements de saxophone: les bruits de clés, qui apparaissent sur le spectrogramme comme une fine colonne verticale "pleine», c'est-à-dire sans traits horizontaux comme les sons périodiques. Ils sont provoqués par le rabattement des clés par les doigts pour boucher les cheminées, c'est-à-dire les trous dans le corps de l'instrument par lesquelles on peut contrôler les hauteurs de notes, ce qui donne un résultat sonore percussif, un claquement bref. Ces sons sont distinctifs puisqu' ils sont 


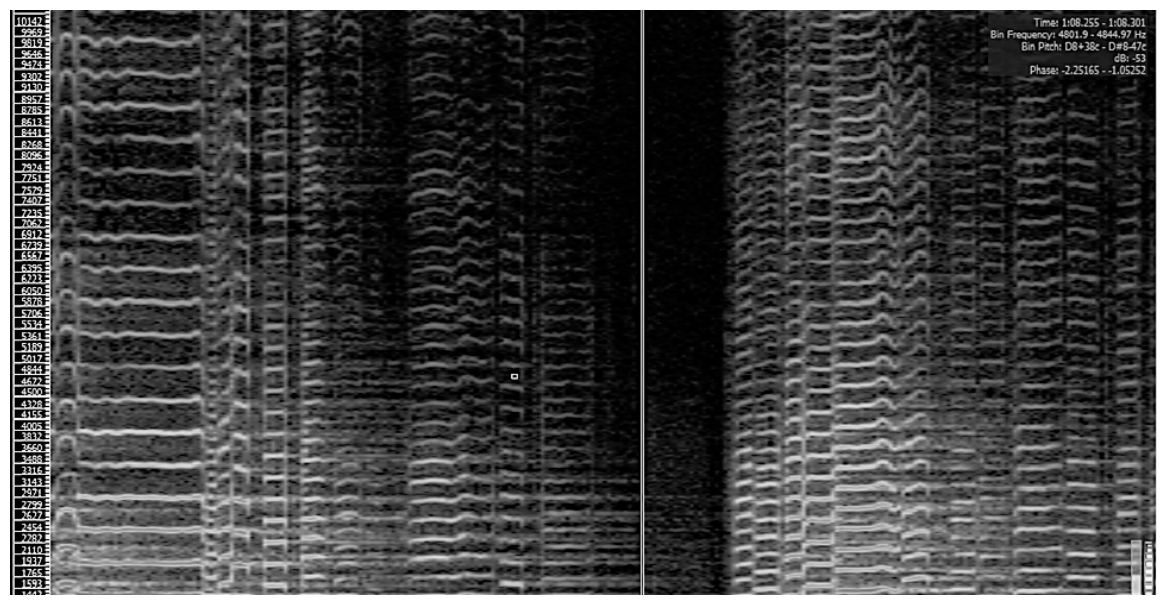

Figure 3. Spectrogramme illustrant l'utilisation systématique du vibrato chez Potter. Les ondulations sont visibles sur plusieurs notes de cet extrait.

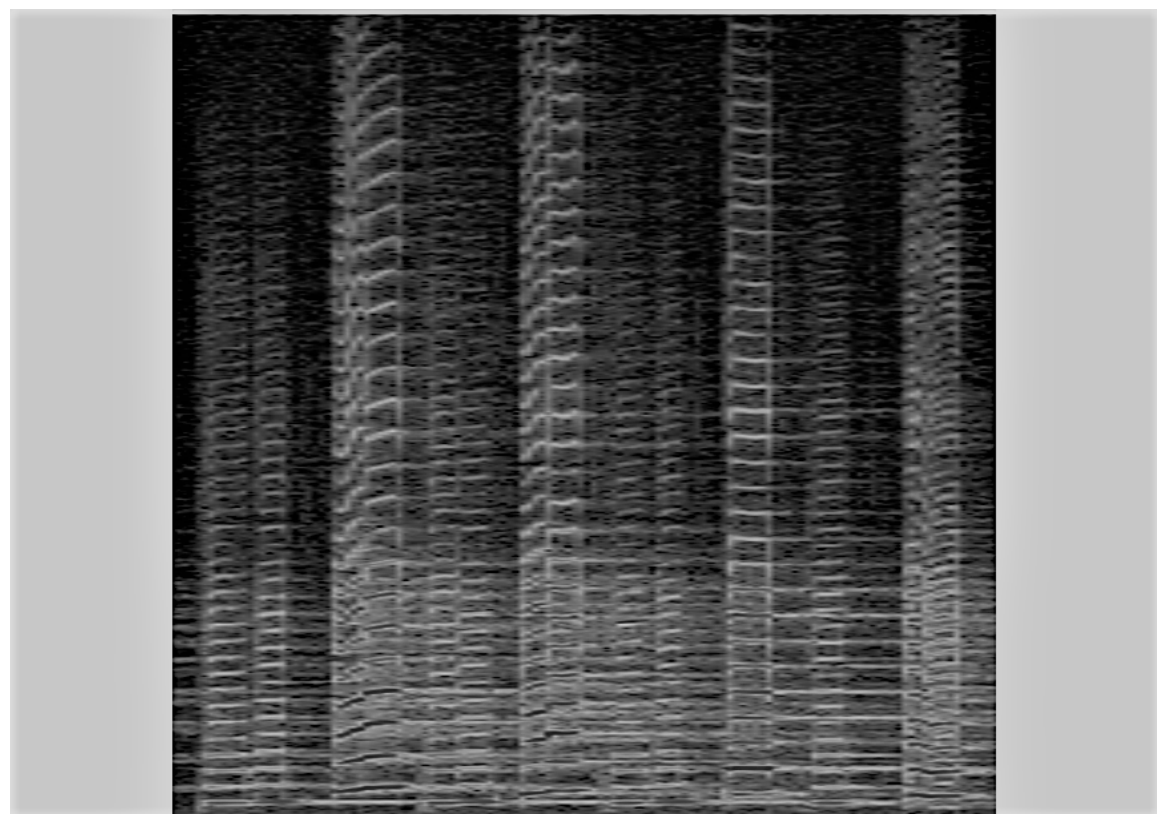

Figure 4. Spectrogramme illustrant l'utilisation de nuances rapides et contrastées chez Potter.

fréquents dans mes improvisations, comme mouvements préparatoires entre deux phrases ou deux notes, tandis qu'ils sont beaucoup moins fréquents chez Potter. Chez ce dernier, ils apparaissent seulement lorsque le bruit de clés est très proche de l'attaque de la note ou lorsque certaines notes sont jouées à bas volume. Il peut y avoir deux explications à la fréquence peu élevée de bruits de clés chez cet improvisateur: soit il rabat les clés en même temps qu'il attaque 


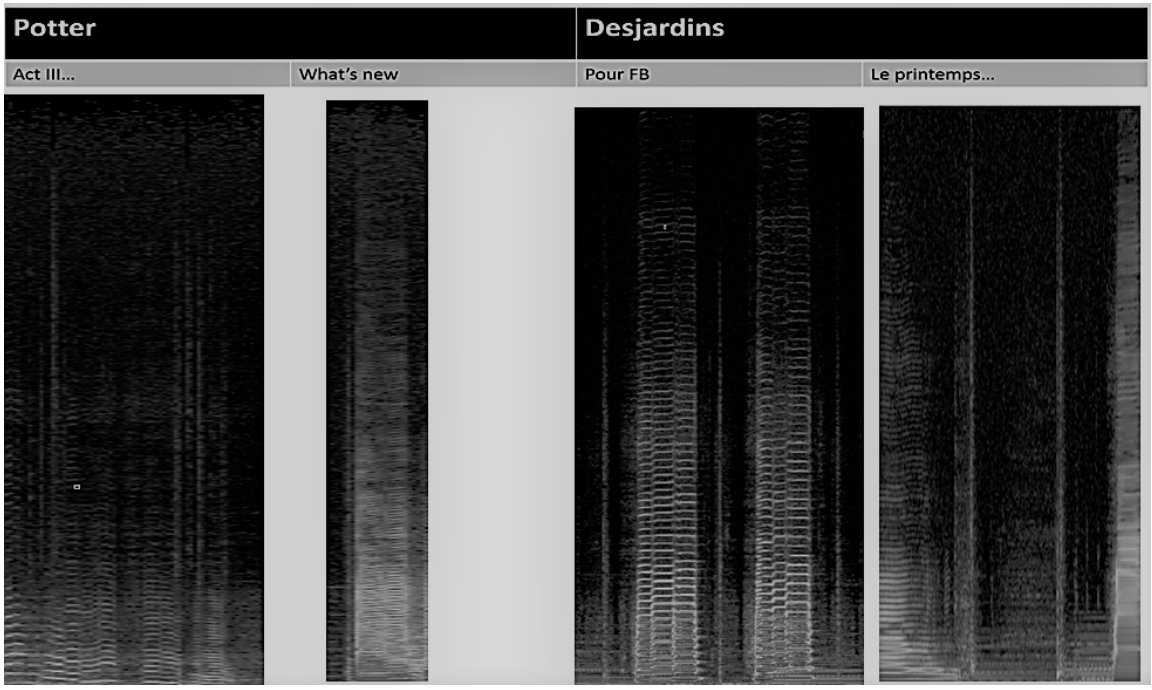

Figure 5. Spectrogramme illustrant les bruits de clés chez Potter et Desjardins.

la note, soit les bruits de clés entendus dans les silences ont été enlevés en postproduction, ce qui relèverait du choix de l'artiste de ne pas faire entendre ces sons. Au final, on observe tout de même que ces sons sont beaucoup plus fréquents dans mon univers sonore que dans celui de Potter.

En conclusion, les traits contingents et constitutifs étant inextricables en improvisation jazz, il était nécessaire d'adopter une façon plus globale de l'analyser, une façon qui considère l'entièreté du sonore, et non seulement les éléments formels ou les effets admis par le genre musical. Comme l'acte de musiquer est l'actualisation d'une pensée par le corps, et que cette actualisation transforme cette pensée en générant un résultat différent de l'intention d'origine, une analyse de la performance la plus exhaustive possible oblige à tenir compte de tout le sonore contenu dans une prestation improvisée. Cela permet une perspective plus incarnée de celle-ci, c'est-à-dire que le corps de l'instrumentiste et ses manifestations sonores sont considérés en tant qu'éléments musicaux à part entière. Les paramètres performanciels étant analysables par l'examen des gestes sonore, ceux-ci permettent d'observer avec plus de précision et de profondeur les pratiques distinctives d'improvisateurs jazz. En effet, les cinq gestes sonores distinctifs que nous avons fait ressortir des improvisations de Chris Potter et des miennes permettent de conclure que ces gestes sonores révèlent des pratiques musicales particulières qui nous distinguent l'un par rapport à l'autre. Bien sûr, un corpus plus vaste nous permettrait d'approfondir cette conclusion en repérant un ensemble de gestes sonores propre à chacun des instrumentistes étudiés. De plus, une analyse exhaustive du sonore demanderait à tenir compte également des paramètres phonographiques (Lacasse 2006) car il est évident à l'écoute que les modalités d'enregistrement et la postproduction ont un impact important sur le son. Dans les cas analysés ici, mes enregistrements 
n'ont pas eu de traitement de postproduction, contrairement à Potter où ils sont apparents, principalement par la présence d'un effet de réverbération.

\section{RÉFÉRENCES}

Béthune, Christian. 1991. «Les enjeux de la technique». Revue d'esthétique 19: 125-131.

Browman, Catherine P., et Louis Goldstein. 1989. "Articulatory gestures as phonological units». Dans Michael Studdert-Kennedy, Status Report on Speech Research, 77-109. New Heaven: Haskins Labs.

Cadoz, Claude, et Marcelo M. Wanderley. 200o. "Gesture - Music». Dans Marcelo Wanderley et Marc Battier, Trends in Gestural Control of Music, 71-93. Paris: Ircam.

Chang, Heewon. 2008. Autoethnography as Method. Walnut Creek: Left Coast Press.

Cottrell, Stephen. 2010. "The rise of phonomusicology». Dans Amanda Bayley, Recorded Music, 15-36. New York: Cambridge University Press.

Cotro, Vincent. 2014. «Le jazz: une mise en jeu particulière du corps». Dans Monique Desroches, Sophie Stévance et Serge Lacasse, Quand la musique prend corps, 363-377. Montréal: Les Presses de l'Université de Montréal.

Cugny, Laurent. 2004. "L'idée de forme dans le jazz». Cahiers de musiques traditionnelles 17: 143-160. http://www.jstor.org/stable/40240522.

-2009. Analyser le jazz. Outre mesure.

Dahl, Sofia, Frédéric Bevilacqua, Roberto Bresin, Martin Clayton, Laura Leante, Isabella Poggi et Nicolas Rasamimanana . 2010. "Gesture in Performance». Dans Rolf Inge Godøy, et Marc Leman, Musical Gestures: Sound, Movement, and Meaning, 36-68. New York: Routledge.

Davidson, Jane. W. 2005. «Bodily Communication in Musical Performance». Dans Dorothy Miell, Raymond MacDonald et David J. Hargreaves, Musical Communication, 215-238. Oxford: Oxford University Press.

Delalande, François. 1988. «La gestique de Gould: éléments pour une sémiologie du geste musical». Dans Ghyslaine Guertin, Glenn Gould Pluriel, 83-111. Québec: Louise Courteau éditrice.

Desroches, Monique. 2008. "Entre texte et performance: l'art de raconter». Cahiers d'ethnomusicologie 21: 103-115. http://ethnomusicologie.revues. org/1215.

- 2014. «Le corps comme signature musicale». Dans Monique Desroches, Sophie Stévance, et Serge Lacasse, Quand la musique prend corps, 287-305. Montréal: Les Presses de l'Université de Montréal.

Doğantan-Dack, Mine. 2011. «In the beginning was gesture: piano touch and the phenomenology of the performing body». Dans Anthony Gritten et Elaine King, New perspectives on music and gesture, 243-265. Farnham: Ashgate.

Genette, Gérard. 1994. L'œuvre de l'art: Immanence et transcendance. Paris: Le Seuil. 
Godøy, Rolf Inge. 2010. "Gestural Affordances of Musical Sound». Dans Rolf Inge Godøy et Marc Leman, Musical Gestures: Sound, Movement, and Meaning, 103-125. New York: Routledge.

- 2011. "Coarticulated gestural-sonic objects in music». Dans Anthony Gritten et Elaine King, New perspectives on music and gesture, 67-82. Farnham: Ashgate.

Héroux, Isabelle et Marie-Soleil Fortier. 2014. «Le geste expressif dans le travail d'interprétation musical». Dans Monique Desroches, Sophie Stévance, et Serge Lacasse, Quand la musique prend corps, 287-305. Montréal: Les Presses de l'Université de Montréal.

Jensenius, Alexander Refsum, Marcelo M. Wanderley, Rolf Inge Godøy, Marc Leman. 2010. "Musical Gestures: concepts and methods in research». Dans Musical gestures: sound, movement, and meaning. Sous la direction de Rolf Inge Godøy et Marc Leman, 12-35. New York: Routledge.

Juslin, Patrick N., et Petri Laukka. 2003. "Communication of emotions in vocal expression and music performance: Different channels, same code? ». Psychological bulletin, 129(5): 770-814.

Kendon, Adam 2004. Gesture: Visible Action as Utterance. Cambridge: Cambridge University Press.

Lacasse, Serge. 2005. «Stratégies narratives dans Stan d'Eminem: Le rôle de la voix et de la technologie dans l'articulation du récit phonographique». Protée 34(2-3): 11-26.

- 2006. "Composition, performance, phonographie: un malentendu ontologique en analyse musicale?». Dans Serge Lacasse et Patrick Roy, Groove. Enquête sur les phénomènes musicaux contemporains, 65-78. Québec: Presses de l'Université Laval.

.2009. «The Phonographic Voice: Paralinguistic Features and Phonographic Staging in Popular Music Singing». Dans Amanda Bayley, Recorded Music: Performance, Culture and Technology, 225-251. New York: Cambridge University Press.

- 2014. «Le corps de la voix qui chante: une relecture phonostylistique de Barthes». Dans Quand la musique prend corps. Sous la direction de Monique Desroches, Sophie Stévance et Serge Lacasse, 107-118. Montréal: Les Presses de l'Université de Montréal.

-2015. "Les performances d'improvisation de Tanya Tagaq: une analyse descriptive de la culture ethno-pop». Itinéraires, 2015-1, mis en ligne le 18 décembre 2015, consulté le 18 décembre 2015. DOI : 10.400o/itineraires.2765

Lortat-Jacob, Bernard. 1987. L'improvisation dans les musiques de tradition orales. Paris: Selaf.

McNeill, David. 2008. Gesture and Thought. Chicago: University of Chicago Press.

Middleton, Richard. 1993. «Popular music analysis and musicology: bridging the gap ». Popular Music 12(2): 177-190.

Mouëllic, Gilles. 2000. Le jazz, une esthétique du XX $X^{e}$ siècle, Rennes: Presses Universitaires de Rennes. 
Nettl, Bruno. et al. Improvisation. Dans Grove Music Online. Consulté à l'adresse: https://doi-org.acces.bibl.ulaval.ca/10.1093/gmo/9781561592630. article.13738.

Nettl, Bruno. 1998. «Introduction: an art neglected by scholarship». Dans Bruno Nettl et Melinda Russell, In the course of performance: studies in the world of musical improvisation, 1-23. Chicago: University of Chicago Press.

Poyatos, Fernando. 1993. Paralanguage: A Linguistic and Interdisciplinary Approach to Interactive Speech and Sound. Amsterdam: John Benjamins.

Rink, John, Neta Spiro, et Nicolas Gold. 2011. «Analysis of Performance». Dans Anthony Gritten et Elaine King, New Perspectives on Music and Gesture, 267-292. Farnham: Ashgate.

Siron, Jacques. 2012. La partition intérieure: jazz, musiques improvisées (8e éd.). Paris: Outre Mesure.

Solomos, Makis. 2013. De la musique au son: l'émergence du son dans la musique des $X X^{e}-X X I^{e}$ siècles. Rennes: Presses universitaires de Rennes.

Sparti, Davide. 2009. "Dans le signe du son. Bruit, voix, corps et improvisation». Images Re-vues 7, mis en ligne le 21 avril 2011, consulté le 25 octobre 2017. http://imagesrevues.revues.org/417.

Stévance, Sophie. 2011a. Musique actuelle. Montréal: Les Presses de l'Université de Montréal.

—_. 2011b. «La beauté libre de la musique actuelle au Québec». Dans Monique Desroches, Territoires musicaux mis en scène, 399-414. Montréal: Presses de l'Université de Montréal.

- 2012. "Une lecture dialogique de l'improvisation libre et collective en musique actuelle et en contact-improvisation». Les Cahiers de la Société québécoise de recherche en musique 13(1-2):117-125.

- 2014. "Tanya Tagaq: l'ethno-pop comme mise en spectacle». Dans Quand la musique prend corps. Sous la direction de Monique Desroches, Sophie Stévance et Serge Lacasse. Montréal 307-328. Les Presses de l'Université de Montréal.

_ _ 2015. «Les performances d'improvisation de Tanya Tagaq: une analyse descriptive de la culture ethno-pop», Itinéraires [En ligne], 2015-1, mis en ligne le 18 décembre 2015, consulté le 18 décembre 2015. DOI: 10.4000/ itineraires.2765.

Stévance, Sophie, et Serge Lacasse. 2017. Research-Creation: Toward a Collaborative Interdiscipline, Routledge.

Tagg, Philip. 2000. «Melody and Accompaniment». Articles for Encyclopedia of Popular Music of the World (EPMOW). https://tagg.org/articles/xpdfs/ melodaccUS.pdf.

Wanderley, Marcelo. M. 1999. «Non-Obvious Performer Gestures in Instrumental Music». Dans A. Braffort, R. Gherbi, S. Gibet et al., Gesture-Based Communication in Human-Computer Interaction, 37-48. Berlin, New York: Springer. 
Wanderley, Marcelo. M., et Philippe Depalle. 2004. «Gestural Control of Sound Synthesis». Engineering and Music - Supervisory Control and Auditory Communication, Proceedings of the EIII 92(4), 632-644.

Windsor, Luke W. 2011. "Gestures in music making: Action, information and perception». Dans Anthony Gritten et Elaine King, New perspectives on music and gesture, 45-66. Farnham: Ashgate.

Wolfe, Joe, Alex. Z. Tarnopolsky, Neville H. Fletcher, Lloyd.C. L. Hollenberg, et John Robert Smith. 2003. "Some effects of the player's vocal tract and tongue on wind instrument sound». Proceedings of the SMAC 3, 307-310.

\section{ENREGISTREMENTS}

Potter, Chris. Act III, scene I. Concord CCD-4843-2. 1998.

Potter, Chris. What's new. Verve 314549 433-2. 2001.

\section{RÉSUMÉ}

Outre le choix des notes, qui est prescrit par notre tradition commune, qu'est-ce qui distingue une ou un improvisateur jazz d'une ou un autre? Qu'est-ce qui constitue leur «son», leur «signature» ? Les méthodes d'analyses plus traditionnelles n'étant pas suffisantes pour circonscrire l'entièreté de l'expression contenue dans l'improvisation jazz, nous devons nous tourner vers l'étude d'enregistrements de solos improvisés afin d'examiner l'aspect «performanciel» de la musique, c'est-à-dire les éléments liés à la production du son par l'instrumentiste. Cependant, l'oreille pouvant omettre certains éléments au profit d'autres, nous avons adapté un modèle d'analyse par spectrogramme utilisé pour l'analyse du chant en musique populaire. En combinant le visuel à l'auditif, ce modèle permet d'effectuer un inventaire plus exhaustif des "gestes sonores» contenus dans les improvisations, c'est-à-dire toutes manifestations sonores produites par des gestes instrumentaux effectués au sein d'un processus musical. À travers un projet de recherche-création, une méthode autoethnographique a permis de distinguer des gestes sonores dans mes propres improvisations et ainsi de les comparer avec celles du saxophoniste de réputation internationale Chris Potter. Les analyses distinguent cinq gestes sonores, subtils mais distinctifs, observés à partir des micro-variations de hauteurs lors de l'attaque ou le déclin de certaines notes, de l'utilisation particulière de sons multiphoniques, du vibrato, des jeux de dynamiques et de bruits de clés.

\section{ABSTRACT}

The choice of music notes as prescribed by our common tradition set aside, what singles out a jazz improviser from another? What are their "sound", their "signature" made of? Considering that traditional methods for analyzing fail to delineate extensively the expressive content of a jazz improvisation, we shall henceforth investigate the recordings of improvised solos looking closely at the performative aspect of music, that is paying attention to details regarding the sonic production by a musical performer. However, considering also that even a careful listening might lead to disregarding some details to the benefit of others, we implemented a spectrographic analysis as it is widely in use for analyzing singing within the field of popular music studies. Combining visual and aural contents, this model allows for a more comprehensive inventory 
of the "sonic gestures" embedded within improvisations, that is of the sonic manifestations produced by instrumental gestures throughout a musical process. Through a research-creation standpoint, an autoethnographic method provided me with a way for discriminating sonic gestures within my own improvisations, and thus compare them to those of internationally acclaimed saxophonist Chris Potter. This analysis has allowed for the identification of five sonic gestures, subtle but distinctive, related to pitch microvariations on the rise and decay of certain music notes, to a specific use of multiphonics, vibrato, sets of dynamics and keystroke noises.

\section{BIOGRAPHIE}

Martin Desjardins est candidat au doctorat en Musicologie (recherche-création) à l'Université Laval, sous la direction de Sophie Stévance. Son projet sur les gestes sonores en tant qu'éléments caractéristiques de l'instrumentiste dans l'improvisation jazz au saxophone ténor lui a permis de décrocher une bourse de trois ans du CRSH en 2015. Également actif en tant que saxophoniste professionnel, il a reçu des bourses de CALQ en 2007 et 2013, en plus d'être jury pour le cet organisme en 2010 et 2014. Enfin, parallèlement à ces projets, il agit comme auxiliaire de recherche et d'enseignement à l'Université Laval. 